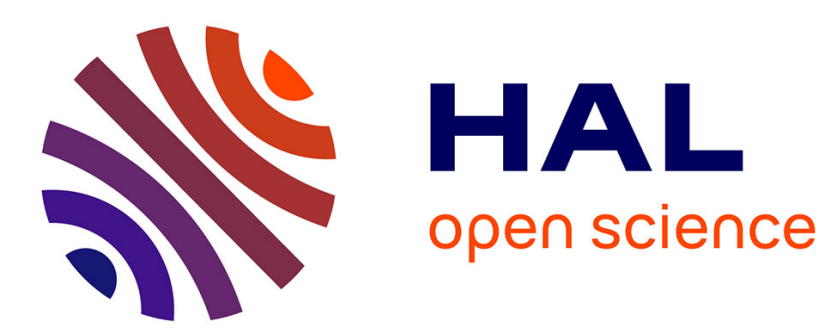

\title{
On the infinitesimal isometries of manifolds with Killing spinors
}

Andrei Moroianu

\section{To cite this version:}

Andrei Moroianu. On the infinitesimal isometries of manifolds with Killing spinors. Journal of Geometry and Physics, 2000, 35, pp.63-74. 10.1016/S0393-0440(99)00079-0 . hal-00126031

\section{HAL Id: hal-00126031 \\ https://hal.science/hal-00126031}

Submitted on 23 Jan 2007

HAL is a multi-disciplinary open access archive for the deposit and dissemination of scientific research documents, whether they are published or not. The documents may come from teaching and research institutions in France or abroad, or from public or private research centers.
L'archive ouverte pluridisciplinaire HAL, est destinée au dépôt et à la diffusion de documents scientifiques de niveau recherche, publiés ou non, émanant des établissements d'enseignement et de recherche français ou étrangers, des laboratoires publics ou privés. 


\title{
ON THE INFINITESIMAL ISOMETRIES OF MANIFOLDS WITH KILLING SPINORS
}

\author{
ANDREI MOROIANU
}

\begin{abstract}
We study the Lie algebra of infinitesimal isometries of 7-dimensional simply connected manifolds with Killing spinors. We obtain some splitting theorems for the action of this algebra on the space of Killing spinors, and as a corollary we prove that there are no infinitesimal isometry of constant length on a 7-dimensional 3Sasakian manifold (not isometric to a space form) except the linear combinations of the Sasakian vector fields.
\end{abstract}

KEYwordS: Infinitesimal isometry, Killing spinor, Sasakian structure

1991 MSC Classification: 53C25, 53A50

\section{INTRODUCTION}

Let $M^{n}$ be a spin manifold and let $\Sigma M$ (resp. $\left.\Sigma^{c} M\right)$ be the fiber bundle of real (resp. complex) spinors. A Killing spinor on $M^{n}$ is a section $\psi$ of $\Sigma M$ satisfying

$$
\nabla_{X} \psi=\lambda X \cdot \psi, \quad \forall X \in T M,
$$

for some real number $\lambda \neq 0$, called the Killing constant of $\psi$. A real Killing spinor gives rise by complexification to a complex Killing spinor, but the converse is not true in general (see below). The existence of a (real or complex) Killing spinor implies that $M$ is an Einstein manifold with Einstein constant $4 \lambda^{2}(n-1)$.

Note that the terminology of real Killing spinors is sometimes used to denote Killing spinors with real Killing constant, in opposition to imaginary Killing spinors, whose Killing constant is imaginary. As in this paper we only consider spinors with real Killing constants, we preferred to use the attribute real in order to describe the nature of the object (as section in a real vector bundle), rather than the nature of its Killing constant.

By rescaling the metric we can always suppose that the Killing constant is equal to $\pm \frac{1}{2}$, and we denote by $N_{ \pm}$the dimension of the complex vector space of complex Killing spinors with Killing constant $\pm \frac{1}{2}$.

Definition 1.1. A compact spin manifold is called of type $N$ if it is simply connected and $N_{+}+N_{-}=N$.

The simply connected manifolds with Killing spinors were described by Bär using the cone construction and the Berger-Simons holonomy theorem (see [1]). In the 
even-dimensional case, the only manifolds of nonzero type are the spheres and the 6-dimensional strictly nearly Kähler manifolds. In this paper we will only consider odd-dimensional simply connected manifolds with Killing spinors, for which we have the following picture (迎):

\begin{tabular}{|l|l|l|}
\hline type of $M$ & $\operatorname{dim}(M)$ & $M$ \\
\hline \hline$N=1$ & 7 & proper weak $G_{2}$-structure \\
\hline$N=2$ & $2 k+1$ & Einstein-Sasakian structure \\
\hline$N=k+2$ & $4 k+3$ & 3-Sasakian structure \\
\hline$N=2^{k}$ & $2 k+1$ & $S^{2 k+1}$ \\
\hline
\end{tabular}

The Lie algebra of infinitesimal isometries of 7-dimensional manifolds of type 1 was studied in [3], where several results about the zero sets of Killing vector fields, as well as a characterization of their exterior derivative in terms of the weak $G_{2}$-structure are obtained.

The aim of this paper is to study the Lie algebra of infinitesimal isometries of manifolds of type 2 and 3 , especially in dimension 7 . Let $\left(M^{2 k+1}, g, \xi\right)$ be a spin manifold of type 2 (i.e. Einstein-Sasakian and not 3-Sasakian). Our first result is that each infinitesimal isometry of $M$ is an infinitesimal automorphism of the Sasakian structure of $M$ (Theorem 2.5). We next describe the action of the Killing vector fields on the set of Killing spinors on $M$, which enables us to prove a splitting theorem for the Lie algebra of the infinitesimal isometries of spin Einstein-Sasakian manifolds (Theorem 2.6). In particular, we prove that the Lie algebra of infinitesimal automorphisms of a weak $G_{2}$-structure on a 7 -dimensional manifold of type 2 is a sub-algebra of codimension 1 in the Lie algebra of infinitesimal isometries. Note that the set of Killing spinors is a vector space if and only if they all have the same Killing constant, which holds exactly when $k$ is odd (cf. [2]).

The situation is more complicated for manifolds of type 3 (3-Sasakian manifolds), since the sub-algebra $I$ spanned by the Sasakian Killing vector fields is no more Abelian. A closer analysis of the action of the Killing vector fields on the space of Killing spinors allows us nevertheless to state a splitting theorem in dimension 7 , and to deduce that $I$ is in fact an ideal of the Lie algebra of infinitesimal isometries for every 7 -dimensional simply connected 3-Sasakian manifold not isometric to $S^{7}$ (Theorem 3.1). As a corollary, we prove that every infinitesimal isometry of constant length on a 7 -dimensional manifold of type 3 is a linear combination (with constant coefficients) of the Sasakian vector fields (Theorem 4.2 and Corollary 4.4).

Acknowledgements. This paper was written while I was member of the Institut für Reine Mathematik of the Humboldt University, supported by the SFB 288 "Differentialgeometrie und Quantenphysik" of the DFG. I would like to thank T. Friedrich for his interest in this work and J. Figueroa-O'Farrill who pointed out several errors in a previous version of this paper. 


\section{MANifolds OF TYPE 2}

The fundamental tool for most of our results is the Lie derivative of spinors with respect to Killing vector fields. We refer the reader to [7] for the definition and the basic properties of this operation. We start by a simple but very useful remark

Lemma 2.1. The Lie derivative with respect to Killing vector fields preserves the space of Killing spinors.

Proof. Recall that the Lie derivative with respect to Killing vector fields satisfies the Leibniz rule for the Clifford product $\left(\right.$ i.e. $\left.\mathcal{L}_{X}(Y \cdot \psi)=\mathcal{L}_{X} Y \cdot \psi+Y \cdot \mathcal{L}_{X} \psi\right)$.

Suppose that $\psi$ satisfies

$$
\nabla_{Y} \psi=a Y \cdot \psi, \forall Y
$$

and let $X$ be a Killing vector field. As $\mathcal{L}_{X}$ preserves the covariant derivative, by taking the Lie derivative with respect to $X$ of the above formula we obtain

$$
\nabla_{\mathcal{L}_{X} Y} \psi+\nabla_{Y}\left(\mathcal{L}_{X} \psi\right)=a \mathcal{L}_{X} Y \cdot \psi+a Y \cdot \mathcal{L}_{X} \psi, \forall Y
$$

so, replacing $Y$ by $\mathcal{L}_{X} Y$ in (11) and subtracting from (2) gives

$$
\nabla_{Y}\left(\mathcal{L}_{X} \psi\right)=a Y \cdot \mathcal{L}_{X} \psi, \forall Y
$$

Definition 2.2. A vector field $\xi$ on a Riemannian manifold $(M, g)$ is called a Sasakian structure if $\xi$ is a Killing vector field of unit length and

$$
\left(\nabla_{X} \varphi\right) Y=g(X, Y) \xi-\eta(Y) X, \quad \text { for all vectors } X, Y \text {. }
$$

In particular, if we take the scalar product with $\xi$ in (3) shows that the tensors $\varphi:=-\nabla \xi$ and $\eta:=g(\xi,$.$) are related by$

$$
\varphi^{2}=-I d+\eta \otimes \xi
$$

Definition 2.3. A triple $\left(\xi_{1}, \xi_{2}, \xi_{3}\right)$ of Sasakian structures is called a 3-Sasakian structure on $M$ if the following conditions are satisfied:

1. The frame $\left(\xi_{1}, \xi_{2}, \xi_{3}\right)$ is orthonormal;

2. For each permutation $(i, j, k)$ of signature $\delta$, the tensors $\varphi_{i}:=-\nabla \xi_{i}$ and $\eta_{i}:=$ $g\left(\xi_{i},.\right)$ are related by $\varphi_{i} \varphi_{j}=(-1)^{\delta} \varphi_{k}+\eta_{j} \otimes \xi_{i}$.

Let $\left(M^{n}, g, \xi\right)$ be a simply connected Einstein-Sasakian manifold and suppose that $M$ is not 3-Sasakian. We first remark that $M$ is spin (see [8]) and admits complex Killing spinors ([四). The space of all Killing spinors on $M$ can actually be constructed explicitly in the following way (see [2])

Lemma 2.4. If $n=1$ or $5 \bmod 8$, then $M$ carries two complex lines of complex Killing spinors with Killing constants $\pm 1 / 2$. Their fiber at each point is given by the solution of the Clifford equation

$$
( \pm 2 \phi(X)+\xi X-X \xi) \psi=0, \forall X
$$


If $n=3$ or 7 mod 8 , then $M$ carries a 2-dimensional complex vector space of complex Killing spinors, with Killing constant $1 / 2$, whose fiber at each point is given by the solution of the Clifford equation

$$
(2 \varphi(X)+\xi \cdot X-X \cdot \xi) \cdot \psi=0, \forall X .
$$

Theorem 2.5. Let $X$ be a Killing vector field on a simply connected Einstein-Sasakian manifold $\left(M^{n}, g, \xi\right)$, and suppose that $M$ is not $3-$ Sasakian. Then $X$ is an infinitesimal automorphism of the Sasakian structure of $M$, that is, $[X, \xi]=0$.

Proof. The spin representations are known to be real for $n=0,6$ and $7 \bmod 8$, complex for $n=1$ and $5 \bmod 8$ and quaternionic for $n=2,3$ and $4 \bmod 8$. In our situation $n$ is odd, and we consider first the case $n=1$ or $5 \bmod 8$. Let $\psi$ be a Killing spinor on $M$ with Killing constant $1 / 2$ and let $X$ be any Killing vector field. Then $\mathcal{L}_{X} \psi$ has the same Killing constant, so

$$
\mathcal{L}_{X} \psi=a \psi
$$

for some complex number $a$. Moreover

$$
\xi \cdot \psi=b \psi
$$

for some $b \in \mathbb{C}$ (because $\xi \cdot \psi$ is a Killing spinor with the same Killing constant, as easily shown by equation (4) after covariant differentiation). Then, taking the Lie derivative with respect to $X$ in (7) and using (6), one obtains $\mathcal{L}_{X} \xi \cdot \psi=0$, so $[X, \xi]=0$.

If $n=7 \bmod 8$, the complex spin bundle has a real structure, so Lemma 2.4 also holds for real Killing spinors in this case. Let $\psi$ be such a spinor. It is clear that $\xi \cdot \psi$ is a Killing spinor, too, and as $M$ is not 3-Sasakian, every real Killing spinor on $M$ is a linear combination of $\psi$ and $\xi \cdot \psi$. Recall that we have an Euclidean scalar product on $\Sigma M$, with respect to which the Clifford multiplication by a vector is skewsymmetric. In particular we have $(X \cdot \phi, \phi)=0$ for all $X$ and $\phi$. Moreover, for every spinor $\phi$ of constant length (so, in particular, for every real Killing spinor), we have $0=X(\phi, \phi)=2\left(L_{X} \phi, \phi\right)$, since the Lie derivation with respect to Killing vector fields preserves the Euclidean product on $\Sigma M$. By Lemma 2.1 we then deduce that $\mathcal{L}_{X} \psi$ and $\mathcal{L}_{X}(\xi \cdot \psi)$ are proportional with $\xi \cdot \psi$ and $\psi$ respectively. Let $a \in \mathbb{R}$ such that $\mathcal{L}_{X} \psi=a \xi \cdot \psi$. We then have

$$
\mathcal{L}_{X}(\xi \cdot \psi)=\mathcal{L}_{X} \xi \cdot \psi+\xi \cdot \mathcal{L}_{X} \psi=\mathcal{L}_{X} \xi \cdot \psi-a \psi
$$

and as the left side of this equation is proportional to $\psi$, the same must be true for $\mathcal{L}_{X} \xi \cdot \psi$, which is actually perpendicular to $\psi$. Thus, $\mathcal{L}_{X} \xi \cdot \psi=0$, which implies $\mathcal{L}_{X} \xi=0$.

Finally, let $n=3 \bmod 8$ and suppose that $n>3$ (for $n=3, M$ would be a space form). In this case the spin bundle is quaternionic and we fix a complex Killing spinor 
$\psi$. It then satisfies (5) and every other complex Killing spinor is of the form $q \psi$, where $q$ is a quaternion. We obtain as before

$$
\xi \cdot \psi=A \psi
$$

for some quaternion $A$ and

$$
\mathcal{L}_{X} \psi=B \psi
$$

for every Killing vector field $X$, where $B$ is a quaternion depending on $X$. Taking the Lie derivative in (8) and using (9) yields

$$
[X, \xi] \psi=[A, B] \psi
$$

We denote $[X, \xi]=Z$ and introduce this in (5) where $Y$ is chosen as to be perpendicular to $Z$ and $\phi(Z)$ (here we use the fact that $\operatorname{dim} M>3$ ). Since $Z$ is perpendicular to $\xi$ (because $\xi$ has constant length) this yields $Z=0$, as claimed.

We now restrict our attention to the 7-dimensional case, and consider a spin manifold $M^{7}$ of type 2 (recall that this means that $M$ is Einstein-Sasakian, but not 3-Sasakian). The existence of a Killing spinor $\psi$ on a 7 -dimensional manifold $M$ is equivalent to that of a weak $G_{2}-$ structure $\omega_{\psi}([3]$, [4]), via the following relation (cf. [2])

$$
\omega_{\psi}(X, Y, \cdot) \cdot \psi=(X \cdot Y+g(X, Y)) \cdot \psi \quad, \quad \forall X, Y \in T M .
$$

By choosing $Y=\xi$ in (10) and using (5) we obtain:

$$
d \xi=2 \xi\lrcorner \omega_{\psi},
$$

Corresponding to the 2-dimensional vector space $\operatorname{Kill}(\Sigma M)$ of Killing spinors, we have a 2-dimensional vector space $G_{2}(M)$ of weak $G_{2}$-structures on $M$, and of course, this vector space is preserved by the action of the infinitesimal isometries, i.e. we have a real representation $l$ of $i(M)$ on $G_{2}(M)$. Our aim is to describe this representation. By Theorem 2.5 we have an exact sequence of Lie algebras

$$
0 \rightarrow \mathbb{R} \xi \rightarrow i(M) \rightarrow i(M) / \mathbb{R} \xi \rightarrow 0 .
$$

Theorem 2.6. (splitting theorem for 7-dimensional spin Einstein-Sasakian manifolds) (i) The restriction of $l$ to $\mathbb{R} \xi$ is isomorphic to the (irreducible) representation of $\mathfrak{u}(1)$ on $\mathbb{C}$.

(ii) There exists a splitting of the above exact sequence in $i(M)=\mathbb{R} \xi \oplus i_{0}(M)$, such that the restriction of $l$ to $i_{0}(M)$ is trivial. 
Proof. (i) We already know that $\mathcal{L}_{\xi} \psi=a \xi \cdot \psi$ for some $a \in R$, and from this it follows that $\mathcal{L}_{\xi}(\xi \cdot \psi)=-a \psi$. All we have to show is that $a$ is nonzero. Suppose that $\mathcal{L}_{X} \psi=0$. Then $X$ is an infinitesimal automorphism of the weak $G_{2}$-structure $\omega_{\psi}$ defined by $\psi$, so by Theorem 6.1 in [3] we obtain:

$$
\left.\pi_{7}(d \xi)=-\frac{2}{3}(\xi\lrcorner \omega_{\psi}\right) .
$$

On the other hand, (11) shows that $d \xi=2 \xi\lrcorner \omega_{\psi} \in \Lambda_{7}^{2} M$, so $\left.\pi_{7}(d \xi)=2 \xi\right\lrcorner \omega_{\psi}$. This, together with (13) would then imply $\xi\lrcorner \omega_{\psi}=0$, so $\xi=0$, a contradiction which proves that $a \neq 0$.

(ii) Let us fix a Killing spinor $\psi$ as before. Consider the linear function $L: i(M) \rightarrow \mathbb{R}$ given by

$$
L(X)=\left(\mathcal{L}_{X} \psi, \xi \cdot \psi\right) .
$$

By the previous discussion, $L$ can be also given by

$$
\mathcal{L}_{X} \psi=L(X) \xi \cdot \psi \text {. }
$$

This formula shows that $L$ is in fact a Lie algebra homomorphism, since

$$
\begin{aligned}
\mathcal{L}_{[X, Y]} \psi & =\left[\mathcal{L}_{X}, \mathcal{L}_{Y}\right] \psi=\mathcal{L}_{X}(L(Y) \xi \cdot \psi)-\mathcal{L}_{Y}(L(X) \xi \cdot \psi) \\
& =-L(X) L(Y) \psi+L(X) L(Y) \psi=0
\end{aligned}
$$

The kernel $i_{0}(M)$ of $L$ is thus an ideal of $i(M)$ of codimension 1. By (i) we see that $i_{0}(M) \cap \mathbb{R} \xi=0$, so $i(M)=\mathbb{R} \xi \oplus i_{0}(M)$. The last statement of the theorem is now trivial, by the very definition of $i_{0}(M)$.

\section{MANifolds OF TYPE 3}

We now consider a 7 -dimensional manifold $M$ of type 3 , i.e. a simply connected 3-Sasakian manifold not isometric with the Euclidean 7-sphere. Denote by $\xi_{i}$ and $\varphi_{i}:=-\nabla_{\xi_{i}}, i \in\{1,2,3\}$, the 3 -Sasakian structure. It is easily seen that the structure group of the frame of oriented orthonormal bundles on $M$ restricts to $S U(2)$, which can be viewed (being simply connected) as a subgroup of $\operatorname{Spin}(7)$, so $M$ is automatically spin. $M$ is also Einstein, by a theorem of Kashiwada ([6]). As before, each Sasakian structure $\xi_{i}$ defines a 2-dimensional sub-bundle of $\Sigma M, H_{i}:=\Phi\left(\xi_{i}\right)$, trivialized by Killing spinors, and conversely, each $H_{i}$ determines $\xi_{i}$. If for some $x \in M$ the fibers $H_{i}(x)$ and $H_{j}(x)$ are equal, then $H_{i}=H_{j}$ (a Killing spinor is determined by its value at any point), so $\xi_{i}=\xi_{j}$, thus implying $i=j$. Consequently, for $i \neq j$ we have $\operatorname{dim}\left(H_{i}(x) \cap H_{j}(x)\right) \leq 1$, so $\operatorname{dim}\left(H_{i}(x) \cup H_{j}(x)\right) \geq 3$. But the space of Killing spinors has dimension 3, so $\operatorname{dim}\left(H_{i}(x) \cup H_{j}(x)\right)=3$, thus showing that $\operatorname{dim}\left(H_{i}(x) \cap H_{j}(x)\right)=1$. We choose Killing spinors of unit norm $\psi_{1}, \psi_{2}, \psi_{3}$ spanning $H_{2} \cap H_{3}, H_{3} \cap H_{1}$ and $H_{1} \cap H_{2}$ respectively. We first show that these three spinors are orthogonal to each other in every point of 
$M$. Let us fix some $x \in M$ and denote by $a_{i j}:=\left(\psi_{i}, \psi_{j}\right)$ in $x$. Using the computation of [2], p.84, we see that $\psi_{1}$ and $\xi_{2} \cdot \psi_{1}$ span $H_{2}$, so we can write

$$
\psi_{3}=a_{13} \psi_{1}+a \xi_{2} \cdot \psi_{1}
$$

for some real number $a$. Similarly we can find $b \in \mathbb{R}$ such that

$$
\psi_{1}=a_{12} \psi_{2}+b \xi_{3} \cdot \psi_{2}
$$

Using (14) and (15) we obtain

$$
\psi_{3}=a_{13} a_{12} \psi_{2}+a_{13} b \xi_{3} \cdot \psi_{2}+a a_{12} \xi_{2} \cdot \psi_{2}+a b \xi_{2} \cdot \xi_{3} \cdot \psi_{2},
$$

and taking the scalar product with $\psi_{2}$ in (16) gives $a_{23}=a_{13} a_{12}$. Similarly we obtain $a_{13}=a_{23} a_{12}$, and hence $a_{23}\left(1-a_{12}^{2}\right)=0$. But $a_{12}^{2}<1$, since otherwise $\psi_{1}= \pm \psi_{2}$, so $a_{23}=0$, and by symmetry, $a_{12}=a_{13}=0$.

We thus have shown that $\psi_{i}$ are everywhere orthogonal to each other, which means that $H_{i}$ is the orthogonal space to $\psi_{i}$ in $H=\cup H_{i}$. Using this and relation (5) we see that $\xi_{2} \cdot \psi_{1}$ lies in $H_{1} \cap H_{2}$, so we can suppose without loss of generality that $\xi_{2} \cdot \psi_{1}=-\psi_{3}$, and similarly $\xi_{3} \cdot \psi_{1}=-\psi_{2}$. We then also have $\xi_{1} \cdot \psi_{2}=\varepsilon \psi_{3}$, where $\varepsilon= \pm 1$. By replacing $\psi_{1}$ with $\varepsilon \psi_{1}$ we finally obtain the homogeneous formulas

$$
\xi_{i} \cdot \psi_{j}=\delta \varepsilon \psi_{k}
$$

where $\delta$ is the signature of the permutation $\{i, j, k\}$.

Let us now compute the Lie derivative of the spinors $\psi_{i}$ with respect to $\xi_{j}$. As $\mathcal{L}_{\xi_{1}}$ preserves $H_{1}, \mathcal{L}_{\xi_{1}} \psi_{3}$ lies in $H_{1}$. Moreover it is orthogonal to $\psi_{3}$, so there is some $a \in \mathbb{R}$ such that $\mathcal{L}_{\xi_{1}} \psi_{3}=a \psi_{2}$. Taking the Lie derivative with respect to $\xi_{1}$ in $\xi_{2} \cdot \psi_{3}=\varepsilon \psi_{1}$ and using the definition of the 3 -Sasakian structure we obtain $\varepsilon \mathcal{L}_{\xi_{1}} \psi_{1}=\xi_{3} \cdot \psi_{3}+a \xi_{2} \cdot \psi_{2}$. This is orthogonal to all of the $\psi_{i}$, as easily follows from (17). On the other hand, $\mathcal{L}_{\xi_{1}} \psi_{1} \in H$ by Lemma 2.1, so $\mathcal{L}_{\xi_{1}} \psi_{1}=0$. This implies that $\xi_{3} \cdot \psi_{3}+a \xi_{2} \cdot \psi_{2}=0$, so $a=-1$ (by (17)) and hence

$$
\mathcal{L}_{\xi_{i}} \psi_{j}=\delta \psi_{k},
$$

where $\delta$ denotes the signature of the permutation $\{i, j, k\}$, with the convention $\delta=0$ if $\{i, j, k\} \neq\{1,2,3\}$.

We are now ready for the main result of this section. As in the Einstein-Sasakian case, there is a natural bijection between the 3-dimensional vector space $H$ of Killing spinors and the set of weak $G_{2}$-structures $G_{2}(M)$, and we consider the real representation $l$ of $i(M)$ on $G_{2}(M)$ given by the Lie derivative via the above bijection. Let $I$ denote the sub-algebra of $i(M)$ spanned by $\xi_{1}, \xi_{2}$ and $\xi_{3}$.

Theorem 3.1. (splitting theorem for 7-dimensional 3-Sasakian manifolds)

(i) The restriction of $l$ to $I$ is isomorphic to the (irreducible) representation of $\mathfrak{s u}(2)=$ $\mathfrak{s o}(3)$ on $\mathbb{R}^{3}$.

(ii) There exists a Lie algebra homomorphism $L: i(M) \rightarrow I$, whose restriction to $I$ is the identity. 
(iii) The restriction of $l$ to $i_{0}(M):=\operatorname{ker}(L)$ is trivial.

(iv) The induced exact sequence of Lie algebras

$$
0 \rightarrow i_{0}(M) \rightarrow i(M) \rightarrow I \rightarrow 0
$$

is splitting. Equivalently, $I$ is an ideal of $i(M)$ and $i(M) \simeq I \oplus i_{0}(M)$.

Proof. (i) Follows directly from (18).

(ii) Given $X \in i(M)$, we denote by $b_{i j}(X)=\left(\mathcal{L}_{X} \psi_{i}, \psi_{j}\right)$, and define $L(X)=$ $\frac{1}{2}\left(b_{23}(X) \xi_{1}+b_{31}(X) \xi_{2}+b_{12}(X) \xi_{3}\right)$. Let us check that $L$ is a Lie algebra homomorphism. Take $X, Y \in i(M)$. We will just show that the $\xi_{1}$-component of $L([X, Y])$ and $[L(X), L(Y)]$ are the same, which is clearly sufficient, by symmetry. From the definition of a 3-Sasakian structure we obtain directly

$$
g\left([L(X), L(Y)], \xi_{1}\right)=\frac{1}{2}\left(b_{31}(X) b_{12}(Y)-2 b_{31}(Y) b_{12}(X)\right) .
$$

Using $\mathcal{L}_{[X, Y]}=\left[\mathcal{L}_{X}, \mathcal{L}_{Y}\right]$ we find

$$
\begin{aligned}
b_{23}([X, Y])= & \left(\mathcal{L}_{X} \mathcal{L}_{Y} \psi_{2}, \psi_{3}\right)-\left(\mathcal{L}_{Y} \mathcal{L}_{X} \psi_{2}, \psi_{3}\right) \\
= & \left(\mathcal{L}_{X}\left(b_{21}(Y) \psi_{1}+b_{23}(Y) \psi_{3}\right), \psi_{3}\right) \\
& -\left(\mathcal{L}_{Y}\left(b_{21}(X) \psi_{1}+b_{23}(X) \psi_{3}\right), \psi_{3}\right) \\
= & b_{21}(Y) b_{13}(X)-b_{21}(X) b_{13}(Y),
\end{aligned}
$$

so finally

$$
g\left(L([X, Y]), \xi_{1}\right)=\frac{1}{2} b_{23}([X, Y])=\frac{1}{2}\left(b_{31}(X) b_{12}(Y)-2 b_{31}(Y) b_{12}(X)\right) .
$$

The last assertion follows from (18).

(iii) If $X \in i_{0}(M)$, then $b_{i j}=0$ so $\mathcal{L}_{X} \psi_{i}$ is orthogonal to $H$. As $\mathcal{L}_{X} \psi_{i} \in H$ (by Lemma 2.1), we obtain $\mathcal{L}_{X} \psi_{i}=0$.

(iv) We have to show that $I$ is an ideal of $i(M)$ or, equivalently, that $I$ and $i_{0}(M)$ are commuting sub-algebras of $i(M)$. Let $X \in i_{0}(M)$. Taking the Lie derivative of (17) with respect to $X$ and using (iii), we see that $\mathcal{L}_{X} \xi_{i} \cdot \psi_{j}=0$, so $\mathcal{L}_{X} \xi_{i}=0$.

\section{INFINITESIMAL ISOMETRIES OF UNIT LENGTH ON 7-DIMENSIONAL MANIFOLDS OF TYPE 3}

As a first application of the above result we have the following

Corollary 4.1. On a 7-dimensional manifold $M$ of type 3, every Killing vector field $X$ of unit length is either a Sasakian structure or an infinitesimal automorphism of the 3-Sasakian structure.

Proof. We decompose $X$ with respect to $i(M)=I \oplus i_{0}(M)$ as $X=A+Y$. From (iv) of the previous theorem we know that $Y$ commutes with the unit Killing vector fields $\xi_{i}$ 
spanning $I$, i.e. it is an infinitesimal automorphism of the 3-Sasakian structure. If $a_{i}$ denotes the $\xi_{i}$-component of $A$, we have to show that either $Y=0$ or $a_{i}=0$. Consider the functions $f_{i}:=g\left(X, \xi_{i}\right)$. As $\left[Y, \xi_{1}\right]=0$, we can compute $\left[X, \xi_{1}\right]=a_{2} \xi_{3}-a_{3} \xi_{2}$, so

$$
\begin{aligned}
0 & =-\frac{1}{2} \mathcal{L}_{\xi_{1}} g(X, X)=-g\left(\mathcal{L}_{\xi_{1}} X, X\right)=g\left(\left[X, \xi_{1}\right], X\right) \\
& =a_{2} f_{3}-a_{3} f_{2},
\end{aligned}
$$

and similarly

$$
a_{2} f_{1}=a_{1} f_{2} \text { and } a_{3} f_{1}=a_{1} f_{3} .
$$

On the other hand, using the definition of the 3-Sasakian structure one easily obtains

$$
\xi_{i}\left(f_{i}\right)=0
$$

and

$$
\xi_{i}\left(f_{j}\right)=2 \delta\left(f_{k}-a_{k}\right),
$$

where $\delta$ denotes as usual the signature of the permutation $\{i, j, k\}$.

Suppose now that at least one of the $a_{i}$ 's doesn't vanish. Without loss of generality, we can suppose $a_{1} \neq 0$. Differentiating in (21) with respect to $\xi_{1}$ and using (22) and (23) we obtain $f_{2}=a_{2}$ and $f_{3}=a_{3}$. Using $\left[Y, \xi_{i}\right]=0$ we find $0=d f_{i}=2 \varphi_{i}(Y)(i=2$ and $i=3$ ), so $Y \in \mathbb{R} \xi_{2} \cap \mathbb{R} \xi_{3}=\{0\}$. It is now clear that $X$ belongs to the 2 -sphere of Sasakian structures of $M$.

Actually a closer analysis of this situation allows us to show that the second case never occurs in the above corollary. Indeed, we have the

Theorem 4.2. Consider an infinitesimal isometry of unit length $X$ on a simply connected 3-Sasakian manifold $\left(M^{7}, g, \xi_{i}\right)$. If $X$ is an infinitesimal automorphism of the 3-Sasakian structure, then $M$ is isometric to the sphere $S^{7}$.

Proof. It is obvious that $\left\{\xi_{1}, \xi_{2}, \xi_{3}, X, \varphi_{1}(X), \varphi_{2}(X), \varphi_{3}(X)\right\}$ is a frame in each point of $M^{7}$ where $X$ doesn't belong to the distribution spanned by the $\xi_{i}$. The set $N$ of such points is obviously open. Suppose that $N$ is not dense in $M$. Then there exists an open subset $U$ of $M$ such that $X$ is a vertical Killing vector field over $U$ (in the sense of Ishihara and Konishi [5]). From Lemma 7 of loc. cit. we obtain that $X$ is a linear combination with constant coefficients of $\xi_{i}$ over $U$, and hence over $M$ since two infinitesimal isometries which coincide on an open set must be equal. This contradicts the fact that $X$ is an infinitesimal automorphism of the 3-Sasakian structure, so we conclude that $N$ is everywhere dense in $M$.

The next step is to prove that in any point of $N$, the sectional curvature of $M$ is constant and equal to 1 . In order to prove this, it suffices to show that

$$
g\left(R\left(Y_{1}, Y_{2}\right) Y_{3}, Y_{4}\right)=g\left(Y_{2}, Y_{3}\right) g\left(Y_{1}, Y_{4}\right)-g\left(Y_{1}, Y_{3}\right) g\left(Y_{2}, Y_{4}\right),
$$


whenever each $Y_{i}$ belong to the frame $\left\{\xi_{1}, \xi_{2}, \xi_{3}, X, \varphi_{1}(X), \varphi_{2}(X), \varphi_{3}(X)\right\}$. First of all, when $Y_{i}=\xi_{j}$ for some $i, j$, then we are done by Lemma 4 at the page 78 in [2]. Actually, this simple lemma just says that the sectional curvature of every 2 -plane containing one of the Sasakian directions is equal to 1.

It is thus enough to check (24) in the case when $Y_{i} \in\left\{X, \varphi_{1}(X), \varphi_{2}(X), \varphi_{3}(X)\right\}$. A priori we would have $4^{4}=256$ possibilities, but thanks to the symmetries of the curvature tensor and of the 3-Sasakian structure, the reader can easily convince himself that it is enough to prove (24) in the following cases:

a) $Y_{1}=\varphi_{1}(X), Y_{2}=Y_{3}=X$

b) $Y_{1}=X, Y_{2}=Y_{3}=\varphi_{1}(X)$

c) $Y_{1}=\varphi_{2}(X), Y_{2}=Y_{3}=\varphi_{1}(X)$

d) $Y_{1}=\varphi_{3}(X), Y_{2}=\varphi_{1}(X), Y_{3}=\varphi_{2}(X)$

e) $Y_{1}=\varphi_{1}(X), Y_{2}=\varphi_{2}(X), Y_{3}=X$

In order to compute the curvature tensor we need the

Lemma 4.3. The following relations hold

$$
\begin{gathered}
\nabla_{X} X=0 ;\left[X, \xi_{i}\right]=0 \quad \forall i \in\{1,2,3\} \\
{\left[X, \varphi_{i}(X)\right]=0 \quad \forall i \in\{1,2,3\}} \\
d f_{i}=2 \varphi_{i}(X) \quad \forall i \in\{1,2,3\} \quad\left(f_{i}=g\left(X, \xi_{i}\right)\right) \\
g\left(X, \varphi_{i}(X)\right)=0 \quad ; \quad g\left(\varphi_{i}(X), \varphi_{j}(X)\right)=\delta_{i j}-f_{i} f_{j} \\
\nabla_{X}\left(\varphi_{i}(X)\right)=\xi_{i}-f_{i} X \quad \forall i \in\{1,2,3\} \\
\nabla_{\varphi_{i}(X)}\left(\varphi_{i}(X)\right)=-2 f_{i} \varphi_{i}(X) \quad \forall i \in\{1,2,3\} \\
\nabla_{\varphi_{i}(X)}\left(\varphi_{j}(X)\right)=-f_{i} \varphi_{j}(X)-f_{j} \varphi_{i}(X)-\delta \xi_{k},
\end{gathered}
$$

where in the last equation, $\delta$ denotes as usual the signature of the permutation $\{i, j, k\}$.

Proof. (25) is just the hypothesis on $X$; (26) follows from the fact that $\mathcal{L}_{X}$ preserves $\xi_{i}$ and $\nabla$, so it preserves $\varphi_{i}$; (27) and (28) are trivial, and (29), (30), (31) follow directly from (26) and the definition of a 3-Sasakian structure. 
We will now check the relation (24) in each of the cases a) - e) using the definition of the 3-Sasakian structure and the above lemma.

a) $R\left(\varphi_{1}(X), X\right) X=-\nabla_{X} \nabla_{\varphi_{1}(X)} X=-\nabla_{X}\left(\xi_{1}-f_{1} X\right)$

$$
=\varphi_{1}(X)=g(X, X) \varphi_{1}(X)-g\left(X, \varphi_{1}(X)\right) X
$$

b) $\quad R\left(X, \varphi_{1}(X)\right) \varphi_{1}(X)$

$$
\begin{aligned}
& =\nabla_{X}\left(-2 f_{1} \varphi_{1}(X)\right)-\nabla_{\varphi_{1}(X)}\left(\xi_{1}-f_{1} X\right) \\
& =-2 f_{1}\left(\xi_{1}-f_{1} X\right)+\varphi_{1}^{2}(X)+f_{1}\left(\xi_{1}-f_{1} X\right)+2 X\left|\varphi_{1}(X)\right|^{2} \\
& =-f_{1}\left(\xi_{1}-f_{1} X\right)-X+\xi_{1} f_{1}+2 X\left|\varphi_{1}(X)\right|^{2} \\
& =X\left|\varphi_{1}(X)\right|^{2} \\
& =g\left(\varphi_{1}(X), \varphi_{1}(X)\right) X-g\left(X, \varphi_{1}(X)\right) \varphi_{1}(X) ;
\end{aligned}
$$

c) $\quad R\left(\varphi_{2}(X), \varphi_{1}(X)\right) \varphi_{1}(X)$

$$
\begin{aligned}
= & \nabla_{\varphi_{2}(X)}\left(-2 f_{1} \varphi_{1}(X)\right)-\nabla_{\varphi_{1}(X)}\left(-f_{2} \varphi_{1}(X)-f_{1} \varphi_{2}(X)+\xi_{3}\right)-2 \nabla_{\xi_{3}}\left(\varphi_{1}(X)\right) \\
= & -2 f_{1}\left(-f_{2} \varphi_{1}(X)-f_{1} \varphi_{2}(X)+\xi_{3}\right)+4 f_{1} f_{2} \varphi_{1}(X)-2 f_{1} f_{2} \varphi_{1}(X)-2 f_{1} f_{2} \varphi_{1}(X) \\
& +2\left(1-f_{1}^{2}\right) \varphi_{2}(X)+f_{1}\left(-f_{2} \varphi_{1}(X)-f_{1} \varphi_{2}(X)-\xi_{3}\right)+\varphi_{3}\left(\varphi_{1}(X)\right)+2 \varphi_{1}\left(\varphi_{3}(X)\right) \\
& -2 f_{3} \xi_{1}+2 f_{1} \xi_{3} \\
= & f_{1} f_{2} \varphi_{1}(X)+\left(2 f_{1}^{2}+2-2 f_{1}^{2}-f_{1}^{2}\right) \varphi_{2}(X)-2 f_{1} \xi_{3}-f_{1} \xi_{3}+\varphi_{2}(X)+f_{1} \xi_{3} \\
& -2 \varphi_{2}(X)+2 f_{3} \xi_{1}-2 f_{3} \xi_{1}+2 f_{1} \xi_{3} \\
= & f_{1} f_{2} \varphi_{1}(X)+\left(1-f_{1}^{2}\right) \varphi_{2}(X) \\
= & g\left(\varphi_{1}(X), \varphi_{1}(X)\right) \varphi_{2}(X)-g\left(\varphi_{1}(X), \varphi_{2}(X)\right) \varphi_{1}(X) ;
\end{aligned}
$$

d) $\quad R\left(\varphi_{3}(X), \varphi_{1}(X)\right) \varphi_{2}(X)$

$$
\begin{aligned}
= & \nabla_{\varphi_{3}(X)}\left(-f_{1} \varphi_{2}(X)-f_{2} \varphi_{1}(X)-\xi_{3}\right)-\nabla_{\varphi_{1}(X)}\left(-f_{2} \varphi_{3}(X)-f_{3} \varphi_{2}(X)+\xi_{1}\right) \\
& +2 \nabla_{\xi_{2}}\left(\varphi_{2}(X)\right) \\
= & 2 f_{1} f_{3} \varphi_{2}(X)+2 f_{2} f_{3} \varphi_{1}(X)-f_{1}\left(-f_{2} \varphi_{3}(X)-f_{3} \varphi_{2}(X)+\xi_{1}\right)-f_{2}\left(-f_{1} \varphi_{3}(X)\right. \\
& \left.-f_{3} \varphi_{1}(X)-\xi_{2}\right)-X+\xi_{3} f_{3}-2 f_{1} f_{2} \varphi_{3}(X)-2 f_{1} f_{3} \varphi_{2}(X) \\
& +f_{2}\left(-f_{1} \varphi_{3}(X)-f_{3} \varphi_{1}(X)+\xi_{2}\right)+f_{3}\left(-f_{1} \varphi_{2}(X)-f_{2} \varphi_{1}(X)-\xi_{3}\right)-X+\xi_{1} f_{1} \\
& +2 f_{2} \xi_{2}-2 f_{2} \xi_{2}+2 X-2 f_{2} \xi_{2} \\
= & f_{2} f_{3} \varphi_{1}(X)-f_{1} f_{2} \varphi_{3}(X) \\
= & g\left(\varphi_{1}(X), \varphi_{2}(X)\right) \varphi_{3}(X)-g\left(\varphi_{2}(X), \varphi_{3}(X)\right) \varphi_{1}(X)
\end{aligned}
$$


e) $\quad R\left(\varphi_{1}(X), \varphi_{2}(X)\right) X$

$$
\begin{aligned}
= & \nabla_{\varphi_{1}(X)}\left(\xi_{2}-X f_{2}\right)-\nabla_{\varphi_{2}(X)}\left(\xi_{1}-X f_{1}\right)+2 \nabla_{\xi_{3}} X \\
= & \varphi_{3}(X)-f_{1} \xi_{2}-f_{2}\left(\xi_{1}-X f_{1}\right)+2 f_{1} f_{2} X+\varphi_{3}(X)+f_{2} \xi_{1}+f_{1}\left(\xi_{2}-X f_{2}\right) \\
& -2 f_{1} f_{2} X-2 \varphi_{3}(X) \\
= & 0=g\left(X, \varphi_{2}(X)\right) \varphi_{1}(X)-g\left(X, \varphi_{1}(X)\right) \varphi_{2}(X) .
\end{aligned}
$$

In order to complete the proof of the theorem, we remark that, $N$ being dense in $M$, the sectional curvature of $M$ has to be constant on the whole $M$, so by simply connectedness, $M$ is isometric to $S^{7}$.

Corollary 4.4. On a 7-dimensional manifold $M$ of type 3, the only Killing vector fields of constant length are the trivial ones, i.e. the linear combinations with constant coefficients of the Sasakian vector fields.

\section{REFERENCES}

[1] C. BÄR, Real Killing spinors and holonomy, Commun. Math. Phys. 154 (1993), 509-521.

[2] H. BAUM, Th. FRIEDRICH, R. GRUNEWALD, I. KATH, Twistor and Killing Spinors on Riemannian Manifolds, Teubner-Verlag, Stuttgart-Leipzig, 1991.

[3] Th. FRIEDRICH, I. KATH, A. MOROIANU, U. SEMMELMANN, On Nearly Parallel $G_{2}{ }^{-}$ Structures, J. Geom. Phys. 23 (1997), 259-286.

[4] A. GRAY, Vector Cross Products on Manifolds, Trans. Amer. Math. Soc. 141 (1969), 465-504.

[5] S. ISHIHARA, M. KONISHI, Fibered Riemannian Spaces with Sasakian 3-Structure, Diff. geom. in honor of K. Yano, Kinokuniya, Tokyo, (1972), 179-194.

[6] T. KASHIWADA, A note on a Riemannian space with Sasakian 3-structure, Nat. Sci. Reps. Ochanomizu Univ. 22 (1971), 1-2.

[7] Y. KOSMANN, Dérivées de Lie des spineurs, Ann. di Matematica ed Appl. 91 (1972), 317-395.

[8] A. MOROIANU, Parallel and Killing Spinors on Spin ${ }^{c}$ Manifolds, Commun. Math. Phys. 187 (1997), 417-428.

A. M. : Centre de Mathématiques, École Polytechnique (umr 7640 du CNRS), Palaiseau, France.

E-mail address: am@math.polytechnique.fr 